\title{
Prevalência de parasitismo intestinal nas aldeias indígenas da tribo Tembé, Amazônia Oriental Brasileira
}

\author{
Prevalence of intestinal parasitism in Tembé tribe indian \\ settlements, Brazilian Eastern Amazon
Rogério dos Anjos Miranda, Fábio Branches Xavier, José Roberto Lima Nascimento e Raimundo Camurça de Menezes

\begin{abstract}
Resumo Para determinar a prevalência de enteroparasitismo nas aldeias Tembé, foi realizado um inquérito coproparasitológico em toda a população (93 índios), em dezembro de 1996. Os parasitos mais freqüentes foram ancilostomídeos (29,0\%), Ascaris lumbricoides (34,4\%), Entamoeba histolytica (12,9\%) e Giardia lamblia (4,3\%). As maiores prevalências de ancilostomídeos e A. lumbricoides foram observadas na aldeia Turé-Mariquita, enquanto que as de E. histolytica e G. Iamblia na Acará-Mirim. Não foi observada diferença significativa sob ponto de vista prático entre a média de idade dos índios parasitados e a dos não parasitados. Sexo esteve relacionado apenas a freqüência de ancilostomídeos, bem maior no sexo masculino. Desse modo, a prevalência de enteroparasitas ainda se encontra elevada para alguns agentes, sugerindo que as medidas de atenção devem ser imediatamente incrementadas a fim de se obterem resultados mais positivos no combate ao enteroparasitismo.
\end{abstract}

Palavras-chaves: Populações indígenas. Parasitismo intestinal. Epidemiologia. Prevalência.

\begin{abstract}
To determine the prevalence of intestinal parasitism in the Tember settlements, parasitological examination was performed in December 1996 in the entire population (93 Indians). Hookworms were found in $29.0 \%$, A. lumbricoides in $34.4 \%$, E. histolytica in $12.9 \%$, and G. lamblia in $4.3 \%$ of the individuals. The Turé-Mariquita settlement had the highest prevalence of hookworms and A. lumbricoides, while Acará-Mirim had the highest prevalence of E. histolytica and G. lamblia. No differences in mean age were found between Indians with parasites and Indians without parasites. The association between intestinal parasites and sex was nonsignificant, except for the prevalence of hookworms, which was very high in males (38.6\%) compared to females (20.4\%). The study showed a high prevalence of some intestinal parasites in the Tembé settlements, indicating that primary and secondary health measures should taken immediately for the prevention of intestinal parasitoses
\end{abstract}

Keys-words: Indian peoples. Intestinal parasitism. Epidemiology. Prevalence.

Núcleo de Medicina Tropical da Universidade Federal do Pará, Belém, PA.

Programa Tembé/FUNAl.

Endereço para correspondência: Dr. Rogério dos Anjos Miranda. R. Vergueiro 415/1205, Liberdade, 01504-001 São Paulo, SP.

Recebido para publicação em 20/1/98. 
A prevalência de parasitoses intestinais é reconhecidamente elevada entre populações ameríndias. Apesar do estabelecimento de medidas de atenção primária e secundária, esta forma de parasitismo muitas vezes se encontra fora de controle por parte dos serviços de saúde, face à grande transmissibilidade dos parasitos, favorecida por fatores de ordem ambiental e sócio-cultural3 41116.

O contato das tribos indígenas da Amazônia com a sociedade nacional é muito variável, desde alguns grupos, em número cada vez mais reduzido, que permanecem em estado de completo isolamento no interior da floresta, até grupos em situação de contato permanente. Esse contato acaba por determinar um maior risco às doenças endêmicas locais ou àquelas introduzidas pela sociedade envolvente, entre as quais se incluem as enteroparasitoses 812 .

O presente trabalho visa determinar a prevalência do parasitismo intestinal nas aldeias indígenas da tribo Tembé, assim como identificar as parcelas da população mais acometidas.

\section{MATERIAL E MÉTODOS}

O grupo indígena Tembé está localizado no município de Tomé-açu, região nordeste do Estado do Pará, a $265 \mathrm{~km}$ de Belém, latitude $2^{\circ} 40^{\prime}-2^{\circ} 41^{\prime}$ e longitude $48^{\circ} 16^{\prime}-48^{\circ} 17^{\prime}$. Está distribuído em três aldeias: Turé-Mariquita, Acará-Mirim e Urumateua. Possui, atualmente, uma população de 93 índios 15.

A língua Tembé está incluída na família Tupiguarani, entretanto, a língua materna não é mais usada no grupo; apenas os homens mais idosos se expressam, ocasionalmente, no dialeto original15.

Trata-se de um estudo transversal realizado nas aldeias Acará-Mirim, Turé-Mariquita e Urumateua, em dezembro de 1996. Neste período, foi efetuado inquérito coproparasitológico por técnicos da FUNAI, utilizando-se amostras de fezes de uma população de 93 índios, 51
$(54,8 \%)$ da aldeia Acará-Mirim, 38 (40,9\%) da Turé-Mariquita e 4 (4,3\%) da Urumateua.

Os métodos utilizados na identificação dos agentes parasitários foram os de sedimentação e exame direto, os únicos exequíveis na ocasião da atividade de campo. Todos os exames foram realizados utilizando-se simultaneamente os dois procedimentos. Os dados são de natureza secundária e as variáveis do estudo correspondem a espécie parasitária, sexo, idade (em anos) e aldeia.

Os dados foram organizados em um gerenciador de banco de dados (Dbase III) e posteriormente processados em um programa de epidemiologia e estatística (Epi Info 6.0).

A inferência estatística não foi necessária em virtude de toda a população ter sido estudada, valendo somente da significância de ordem prática para as afirmações científicas.

\section{RESULTADOS}

De acordo com os resultados, ancilostomídeos foram encontrados em 27 (29\%), A. lumbricoides em $32(34,4 \%)$, Trichiura trichiura em $1(1,1 \%)$, não tendo sido encontrados no estudo índios parasitados com Strongyloides stercoralis, Taenia sp e Enterobius vermicularis. Em relação aos protozoários, E. histolytica foi encontrada em $12(12,9 \%)$ e em $4(4,3 \%)$ G. lamblia (Tabela 1$).$
As maiores prevalências de ancilostomídeos e A. lumbricoides foram observadas na aldeia Turé-Mariquita, respectivamente, 42,1 e $44,7 \%$, enquanto que as de E. histolytica (17,6\%) e G. lamblia $(5,9 \%)$ na Acará-Mirim. Em relação aos quatro índios da aldeia Urumateua, apenas 1 apresentou parasitismo intestinal, sendo por ancilostomídeos (Tabela 1).

Tabela 1 - Prevalência de enteroparasitas em indígenas da tribo Tembé, dezembro de 1996.

\begin{tabular}{|c|c|c|c|c|}
\hline \multirow[t]{2}{*}{ Enteroparasitas } & \multicolumn{3}{|c|}{ Aldeias } & \multirow{2}{*}{$\begin{array}{c}\text { Total } \\
\mathrm{N}=93 \%\end{array}$} \\
\hline & $\begin{array}{l}\text { Acará-Mirim } \\
(\mathrm{N}=51) \%\end{array}$ & $\begin{array}{l}\text { Turé-Mariquita } \\
\qquad(\mathrm{N}=38) \%\end{array}$ & $\begin{array}{l}\text { Urumateua } \\
(\mathrm{N}=4) \%\end{array}$ & \\
\hline Ancilostomídeos & 19,6 & 42,1 & 25,0 & 29,0 \\
\hline A. lumbricoides & 29,4 & 44,7 & - & 34,4 \\
\hline T. trichiura & 1,9 & - & - & 1,1 \\
\hline E. histolytica & 17,6 & 7,9 & - & 12,9 \\
\hline G. lamblia & 5,9 & 2,6 & - & 4,3 \\
\hline
\end{tabular}


Com relação a idade, a prevalência de ancilostomídeos e de $A$. lumbricoides foi significativamente maior na faixa etária que vai dos 10 aos 14 anos, respectivamente, 63,6 e $63,3 \%$. Já os protozoários, E. histolytica e
G. lamblia, que em geral apresentaram menores freqüências do que os helmintos, estiveram ausentes nos grupos de 0 a 1 e 5 a 9 anos para E. histolytica, e nos de 1 a 9 para G. lamblia (Tabela 2).

Tabela 2 - Prevalência (\%) de enteroparasitas nas aldeias Tembé de acordo com idade e sexo, e mediana de idade dos parasitados e não-parasitados por cada agente. Dezembro de 1996.

\begin{tabular}{lccccc}
\hline Idade (anos) & $\mathrm{N}$ & Ancilostomídeos & A. lumbricoides & E. histolytica & G. lamblia \\
\hline$<1$ & 13 & 7,7 & 7,7 & - & 7,7 \\
$1-4$ & 14 & 21,4 & 28,5 & 14,3 & - \\
$5-9$ & 13 & 30,7 & 38,4 & - & - \\
$10-14$ & 11 & 63,6 & 63,3 & 18,2 & 9,1 \\
$\geq 15$ & 42 & 28,5 & 35,7 & 19,0 & 4,8 \\
Parasitados & $12,0(0,4-58,5)^{*}$ & $13,6(0,4-58,5)$ & $16,7(3,3-53,9)$ & $19,1(0,7-27,8)$ & \\
Não parasitados & $14,0(0,1-68,8)$ & $13,8(0,1-68,8)$ & $12,1(0,1-68,8)$ & $13,7(0,1-68,8)$ & \\
Sexo & 44 & & & & 13,6 \\
$\quad$ Masculino & 49 & 38,6 & 32,0 & 12,2 & 2,3 \\
$\quad$ Feminino & 20,4 & 36,7 & & 6,1 \\
\hline * mediana (limites mínimo-máximo) & & & &
\end{tabular}

Não foi observada diferença significativa sob ponto de vista prático entre a média de idade dos índios parasitados e a dos não parasitados. Também não foram significativas as diferenças entre prevalências de enteroparasitas por sexo, exceto a prevalência de ancilostomídeos, significativamente maior no sexo masculino, $38,6 \%$ contra os $20,4 \%$ do sexo feminino.

\section{DISCUSSÃO}

As enteroparasitoses, nas aldeias Tembé, ainda são de elevada prevalência para alguns agentes. Inquéritos coproparasitológicos têm também demonstrado elevada ocorrência de parasitismo intestinal em outras tribos indígenas do Brasil3 11 13. Entretanto, diversos autores têm também descrito baixas prevalências em comunidades indígenas, enfatizando a importância do uso de medicação em massa no controle desta forma de parasitismo4 816 .

Vários fatores são responsáveis pela elevada prevalência de parasitismo nestas aldeias. Alguns fatores que favorecem a transmissão dos agentes parasitários têm sido observados, tais como o destino inadequado dos dejetos, a ingestão de água sem devido tratamento, proveniente de mananciais localizados às proximidades das aldeias, e ausência do uso de calçados por boa parte da população, principalmente entre as crianças. Não há estudos sobre hábitos dietéticos nas aldeias, embora se tenha observado com freqüência o consumo de carne bovina e suína durante as visitas de campo. Fatores ambientais não devem ser esquecidos, o clima úmido e o solo arenoso da região propiciam o desenvolvimento e manutenção de estágios infectantes destes parasitas. As evidências de fatores genéticos e imunológicos na determinação de susceptibilidade ainda são pouco esclarecidas e, portanto, não podem ser afastadas 6 .

As espécies A. lumbricoides e E. histolytica foram os helmintos e protozoários de maior prevalência, respectivamente. Estudos em comunidades indígenas e não-indígenas têm apresentado diversos resultados, sendo A. lumbricoides e Ancilostomídeos os helmintos, e E. histolytica os protozoários de maior prevalência2 3591013 14. Vale salientar, também, a alta prevalência de $E$. histolytica em aldeias indígenas da Amazônia, como a dos Parakanã, sudeste do Estado do Pará14, alertando as equipes de saúde para o risco de formas invasivas intestinais e extra-intestinais de amebíase ${ }^{1}$.

$\mathrm{Na}$ identificação dos agentes parasitários, os métodos de sedimentação espontânea e exame direto foram os únicos métodos disponíveis nas aldeias. Limitações acerca de sensibilidade e especificidade dos referidos métodos são aqui 
enfatizadas como causa provável de vícios de informação, possivelmente, de resultados falso negativos para as espécies $E$. vermicularis, Taenia sp. e S.stercoralis. Ainda que presente, trata-se de um viés igualmente distribuído nos resultados, haja visto todas as amostras fecais terem sido examinadas pelo dois métodos simultaneamente.

Apesar do estudo não ter sido projetado com finalidade de evidenciar elos causais, como seria num estudo caso-controle, não se observou diferença significativa sob ponto de vista prático entre as médias de idade dos índios parasitados e dos não parasitados para cada agente. Maior prevalência de parasitismo por ancilostomídeos e A. lumbricoides foi encontrada entre as crianças de 10 a 14 anos. Estudos em populações nãoindígenas têm evidenciado maior freqüência de parasitoses em faixas etárias menores, especialmente entre as crianças de baixo nível sócio-econômico 710 17. Já estudos em populações indígenas têm ressaltado alguns fatores possivelmente envolvidos na maior transmissão dos enteroparasitas entre as crianças, tais como a ausência do uso de calçados por grande parte delas e o maior contato das mesmas com o solo contendo formas infectantes. O hábito de defecar dentro e nas proximidades do domicílio foi outro fator identificado em comunidades indígenas, embora relacionado a todas as faixas de idade ${ }^{14}$.
No presente estudo, não foi verificada diferença substancial entre as prevalências de enteroparasitas em relação a sexo, exceto para os ancilostomídeos, bem mais freqüente no sexo masculino. Ressalta-se a existência de relato de maior freqüência de poliparasitismo em crianças não-índias do sexo masculino, fato relacionado pelos autores ao maior contato com o solo contendo formas parasitárias infectantes ${ }^{7}$. $\mathrm{Na}$ realidade dos Tembé, as diferenças de atividade entre os sexos, fato que somente se observa a partir de uma certa faixa etária, necessitam de estudo mais profundo para que se possam evidenciar elos causais.

Vale salientar, ainda, as conseqüências negativas do enteroparasitismo para a qualidade de vida e bem-estar desta população, assim como o risco de quadros graves, processos obstrutivos e formas invasivas, que devem ser considerados pela equipe de saúde atuante nas aldeias.

Desse modo, o presente estudo demonstra que a prevalência de alguns parasitas intestinais se encontra elevada nas aldeias Tembé. A realização periódica de inquéritos coproparasitológicos, incluindo a determinação da carga parasitária, faz-se necessária para o controle das enteroparasitoses nas aldeias, assim como para a avaliação das estratégias e medidas de saúde a serem implementadas no combate a esta forma de parasitismo.

\section{AGRADECIMENTOS}

À coordenadora do subprograma de educação, Célia Valois, e aos técnicos do Programa Tembé/FUNAI, pela amizade e indispensável colaboração na realização deste trabalho.

\section{REFERÊNCIAS BIBLIOGRÁFICAS}

1. Andrade DR, Júnior DRA. Amebíase. In: Veronesi $R$, Foccacia R (ed) Tratado de Infectologia, $1^{\text {a }}$ edição, Atheneu, São Paulo, p.1149-1159, 1996

2. Cardoso GS, Santana ADC, Aguiar CP. Prevalência e aspectos epidemiológicos da giardíase em creches no Município de Aracaju, SE, Brasil. Revista da Sociedade Brasileira de Medicina Tropical 28:25-31, 1995.

3. Coimbra CEA, Melo DA. Enteroparasitas e Capillaria sp. entre o grupo Suruí, Parque Indígena Aripuanã, Rondônia. Memórias do Instituto Oswaldo Cruz 76:299-302, 1981.

4. Coimbra CEA, Santos RV. Parasitismo intestinal entre o grupo indígena Zoró, Estado do Mato Grosso (Brasil). Cadernos de Saúde Pública 7:100-103, 1991.

5. Collins RF, Edwards LD. Prevalence of intestinal helminths and protozoans in a rural population segment of the Dominican Republic. Transactions of the Royal
Society of Tropical Medicine and Hygiene 75:549-551, 1981.

6. Cooper PJ, Guevara A, Guderian RH. Intestinal helminthiases in Ecuador: the relationship between prevalence, genetic, and socioeconomic factors. Revista da Sociedade Brasileira de Medicina Tropical 26:175180, 1993.

7. Dórea RC, Salata E, Padovani CR, Anjos GL. Control of parasitic infections among school children in the peri-urban area of Botucatu, São Paulo, Brazil. Revista da Sociedade Brasileira de Medicina Tropical 29:425-430, 1996.

8. Ferrari JO, Farreira MU, Camargo LMA, Ferreira CS. Intestinal parasites among Karitiana indians from Rondônia State, Brazil. Revista do Instituto de Medicina Tropical de São Paulo 34:223-225, 1992.

9. Gonçalves JF, Tanabe M, Medeiros FP, Gonçalves FJ, Aca IS, Motta SR, Tateno S, Takeuchi T. Parasitological 
and serological studies on amoebiasis and other intestinal parasitic infections in the rural sector around Recife, Northeast Brazil. Revista do Instituto de Medicina Tropical de São Paulo 32:428-435, 1990.

10. Kobayashi J, Hasegawa H, Forli AA, Nishimura NF, Yamanaka A, Shimabukuro T, Sato Y. Prevalence of intestinal parasitic infection in five farms in Holambra, São Paulo, Brazil. Revista do Instituto de Medicina Tropical de São Paulo 37:13-18, 1995.

11. Lawrence DN, Neel JV, Abadie SH, Moore LL, Adams LJ, Healy GR, Kagan IG. Estudos epidemiológicos entre populações ameríndias da Amazônia. Parasitoses intestinais em povoações recentemente contactadas e em aculturação. Acta Amazonica 13:393-407, 1983.

12. Martins SJ, Menezes RC. Nutritional status of children under 5 years of age in indian villages of the Parakanã tribe in Brazil's eastern Amazônia. Revista de Saúde Pública 28:1-8, 1994.

13. Miranda RA, Xavier FB, Menezes RC. Parasitismo intestinal e desnutrição protéico-energética em crianças menores de 10 anos de idade de duas aldeias indígenas na tribo Parakanã, sudeste do estado do Pará - Brasil. Arquivos brasileiros de Pediatria 4:169-173, 1997.

14. Miranda RA, Xavier FB, Menezes RC. Parasitismo intestinal em uma aldeia indígena Parakanã no sudoeste do Estado do Pará, Brasil. Cadernos de Saúde Pública 14:507-511, 1998.

15. Povos Indígenas do Brasil. CEDI, São Paulo, 1985.

16. Santos RV, Coimbra CEA, Flowers NM, Silva JP. Intestinal parasitism in the Xavánte indians, Central Brazil. Revista do Instituto de Medicina Tropical de São Paulo 37:145-148, 1995.

17. Shetty N, Narasinha M, Raghuveer TS, Elliot E, Farthing MJG, Macaden R. Intestinal amoebiasis and giardiasis in southern indian infants and children. Transactions of the Royal Society of Tropical Medicine and Hygiene 84:382384, 1990. 